\title{
Cognitive Bias Modification for Addictive Disorders: Emerging Knowledge and Persistent Gaps
}

\author{
Melvyn WB Zhang ${ }^{1,2, *}$ and Helen E. Smith ${ }^{2}$ \\ 1 National Addiction Management Service, Institute of Mental Health, Singapore 539747, Singapore \\ 2 Family Medicine and Primary Care, Lee Kong Chian School of Medicine, Nanyang Technological \\ University Singapore, Singapore 308232, Singapore; h.e.smith@ntu.edu.sg \\ * Correspondence: melvynzhangweibin@gmail.com
}

Received: 18 September 2020; Accepted: 23 October 2020; Published: 1 November 2020

\begin{abstract}
The advances in experimental psychology have led to the discovery of unconscious, automatic biases (attentional and approach biases) that account for the lapse and relapses amongst individuals with addictive disorders. To date, there has been extensive investigation into the assessment and modification of attention biases amongst individuals with the highly prevalent substance use disorders. Alternative modalities, such as technology, has also been evaluated for the delivery of these interventions. Given this, the specific objectives of this perspective article are in highlighting the emerging knowledge that has accrued over the last three years, and in addressing several of the research gaps in the previous article. Firstly, this perspective article will examine the evidence for biases and bias modification amongst the highly prevalent substance use disorders. This article will also examine how such bias modification has been utilized clinically. Secondly, this perspective article will also examine how technology has been applied to these bias modification interventions. Thirdly, this article will also examine the literature to highlight the biological underpinnings following bias modification. Fourthly, this article also examines the limitations of existing bias modification paradigms and methods taken to better these conventional interventions. The article concludes with highlighting the outstanding gaps in the current knowledge. To date, there remains mixed evidence for the modification of attention biases; and there are a paucity of studies examining the effectiveness of mobile delivery of bias intervention. Only one study has considered patients' perspective in the development of an intervention. There remained several gaps in the knowledge, which future research could address.
\end{abstract}

Keywords: attention bias; cognitive bias; psychiatry

\section{Introduction}

In recent years, the advances in experimental psychology have led to the discovery of unconscious, automatic biases (attentional and approach biases) that account for the lapses and relapses amongst individuals with addictive disorders. Attentional and approach biases are unconscious, relatively automatic processes that result in individuals' attention being allocated towards substance-related cues, or having response tendencies (for example, that of actions) towards such cues.

With regards to the evidence for the existence of these biases, several meta-analytical reviews have provided evidence, such as that of MacLean R.R. et al. (2018) [1], who reported that individuals diagnosed with opioid use disorder have a greater magnitude of attentional biases, as compared to controls; and that of $\mathrm{O}^{\prime} \mathrm{Neill}$ et al. (2020) [2], which considered 14 independent studies comprising a total of 1271 participants with cannabis use disorders and reported that cannabis users have more significant attentional biases as compared to healthy controls [2]. Other reviews, such as that by Cristea et al. (2016) [3] have also investigated the effectiveness of bias modification amongst individuals 
with addictive disorders. In their prior review, which was limited to the inclusion of participants with alcohol and tobacco use disorders, they reported that bias modification had a moderate effect size of hedges G 0.60 for cognitive bias. Unfortunately, Cristea et al. (2016)'s [3] review was criticized for its methodology, and Boffo M et al. (2019) [4] have since advanced the work and further examined the effectiveness of bias modification, by undertaking a Bayesian network meta-analysis. Boffo M et al. (2019) [4] reported the magnitude of bias modification for cognitive biases to be 0.23 when investigated amongst a sample of participants with alcohol and tobacco use disorders.

These prior reviews have provided justification for the existence of biases amongst individuals with addictive disorders and examined the effectiveness of bias modification. Unfortunately, most of these prior reviews are limited to the examination of cognitive biases amongst individuals with either alcohol or tobacco disorders. Substance disorders, that of opioid use, cannabis use and stimulant use disorders are increasingly prevalent globally. According to the recent statistics released by the United Nations Office of Drugs and Crime, cannabis was the most widely abused substance in 2018, with an estimated 192 million individuals abusing it globally [5]. Substance like opioids are still widely abused, and this has resulted in significant mortality [5]. It is timely now to examine the literature, to determine if there have been further evaluations of cognitive bias assessment and modification amongst these highly prevalent substance disorders. The delivery of cognitive bias modification has been transformed in recent years, along with the advances in technologies. Both web-based and mobile technologies have been used for the delivery of these interventions.

The advances in cognitive bias modification might have resulted in new knowledge, with resultant clinical and research implications for the field of addiction psychiatry, as aforementioned. Zhang et al. (2018) [6], in their previous perspective article that was published three years ago, have reviewed the evidence for attentional bias modification; this includes the effects of pharmacological drugs on attentional biases and briefly overviewed the literature of how technological tools have been applied. The specific objectives of this perspective article are in highlighting the emerging knowledge that has accrued over the last three years, and in addressing several of the research gaps in the previous article. Firstly, this perspective article will examine the evidence for biases and bias modification amongst the highly prevalent substance use disorders. This article will also examine how such bias modification has been utilized clinically. Secondly, this perspective article will also examine how technology has been applied to these. Thirdly, this article will also examine the literature to highlight the biological underpinnings following bias modification. Fourthly, this article also examines the limitations of existing bias modification paradigms and methods taken to better these conventional paradigms. The article concludes with highlighting the outstanding gaps in the current knowledge.

\section{Evidence for Bias Modification Amongst Highly Prevalent Substance Disorders}

Zhang et al. (2018) [7,8] conducted a series of systematic reviews and reported that cognitive biases, both approach and attentional biases were consistently present amongst their identified studies. Their identified studies included 11 studies involving participants with opioid use disorders, 16 studies with participants with cocaine use disorders, nine studies with participants with cannabis use disorders and two studies with participants with different disorders (i.e., participants with polysubstance use disorders). Unfortunately, they did not manage to determine the effectiveness of bias modification, as the reported outcomes in each of the identified studies were heterogeneous. Since the publication of these reviews, more recently, MacLean R.R. et al. (2018) [1] conducted a meta-analysis of attentional biases amongst individuals with opioid use disorder and reported that individuals with opioid use disorder have a robust attentional bias, as compared to controls. Attentional biases were also present amongst individuals who were maintained on opioid substitution therapy, such as methadone maintenance. This implies that medications, like methadone, which could keep individuals in abstinence, might not have managed to attenuate underlying automatic biases; and these individuals could still be liable to further slips and relapses. For cannabis use disorders, O'Neill et al. (2020) [2], 
in their systematic review and meta-analysis, considered 14 manuscripts that included a total of 1271 participants with cannabis use disorders (cannabis users $n=1044$; controls, $n=107$ ). They reported that cannabis users have greater attentional biases towards cannabis cues, as compared to healthy controls. The evidence also suggests that the magnitude of the attentional biases was greater when the duration of the presentation of the stimulus was shorter. These studies, however, have not managed to synthesize the evidence for the effectiveness of bias modification. Hence, there has not been any concrete knowledge as to whether bias modification works for individuals with cannabis use disorders.

Hietmann et al. (2018) [9], in their review, attempted to examine the effectiveness of bias modification. Of the eighteen studies they identified, most of the studies were interventions for individuals with alcohol or tobacco use disorders; but three studies had included participants with opioid use disorders. Of their included studies for opioid use disorders, only one (Ziaee et al., 2016) [10] provided evidence that drug attention control training helped in the reduction of biases. More recently, there has been a further evaluation of bias modification amongst individuals with stimulant use (cocaine use) disorders and methamphetamine use disorders. Mayer A.R. et al. (2016) [11], in their prior study, examined how bias modification training affected brain activity and drug use. Amongst their sample of 37 participants, the authors did not find that the intervention moderated the use of cocaine. Dean A.C. et al. (2019) [12] examined attentional bias modification amongst 42 methamphetamine-dependent clients who were receiving residential treatment for their drug use. They reported that bias retraining did not result in a reduction in attentional biases and that there was no impact of bias retraining on cravings. Whilst these studies have presented generally negative findings, Manning V. et al. (2019) [13] examined a computerized approach bias modification intervention amongst patients with methamphetamine use disorders. They reported that their participants accepted the intervention and that the intervention was feasible, with abstinence rate at $61 \%$ at two weeks and $54 \%$ at three months [13]. It is thus clear from these studies that cognitive biases (both approach and attentional biases) are present in highly prevalent substance use disorders. However, the evidence for bias modification remains mixed.

There are several reasons that could have accounted for these results. The actual effectiveness of bias modification could have been undermined in some prior reviews (such as that of Cristea et al., 2016) [3], as they have synthesized the findings from both clinical as well as experimental laboratory-based studies (Wiers et al., 2018) [14]. Wiers et al. (2018) [14] in their commentary estimated the effect size of cognitive bias modification, based on a prior large-scale published study by Eberl et al. (2013), and ascertained that cognitive bias modification results in at least $8.5 \%$ less relapse as compared to no intervention. Drawing parallel with what Wiers et al. (2018) [14] have commented previously, the lack of effectiveness in some of the identified studies, as aforementioned might be due to the sample size, and the resultant lack of adequate power. More importantly, Wiers et al. (2018) [14] has highlighted that the results could also be clouded by the nature of the tasks used for the assessment and measurement of biases. These issues should be taken into consideration when researchers are conceptualizing future interventions involving the evaluation of cognitive bias modification interventions amongst substance users.

It is, however, heartening to learn that there has been a further evaluation of cognitive bias modification amongst clinical samples. For example, Dean A.C. et al. (2019) [12] have examined it amongst methamphetamine-dependent individuals who were in residential treatment; and similarly, Manning V. et al. (2019) [13] have also examined it amongst methamphetamine-dependent individuals recruited from inpatient withdrawal units. Table 1 provides an overview and summary of the studies we have discussed. 
Table 1. Overview and Summary of Main Findings.

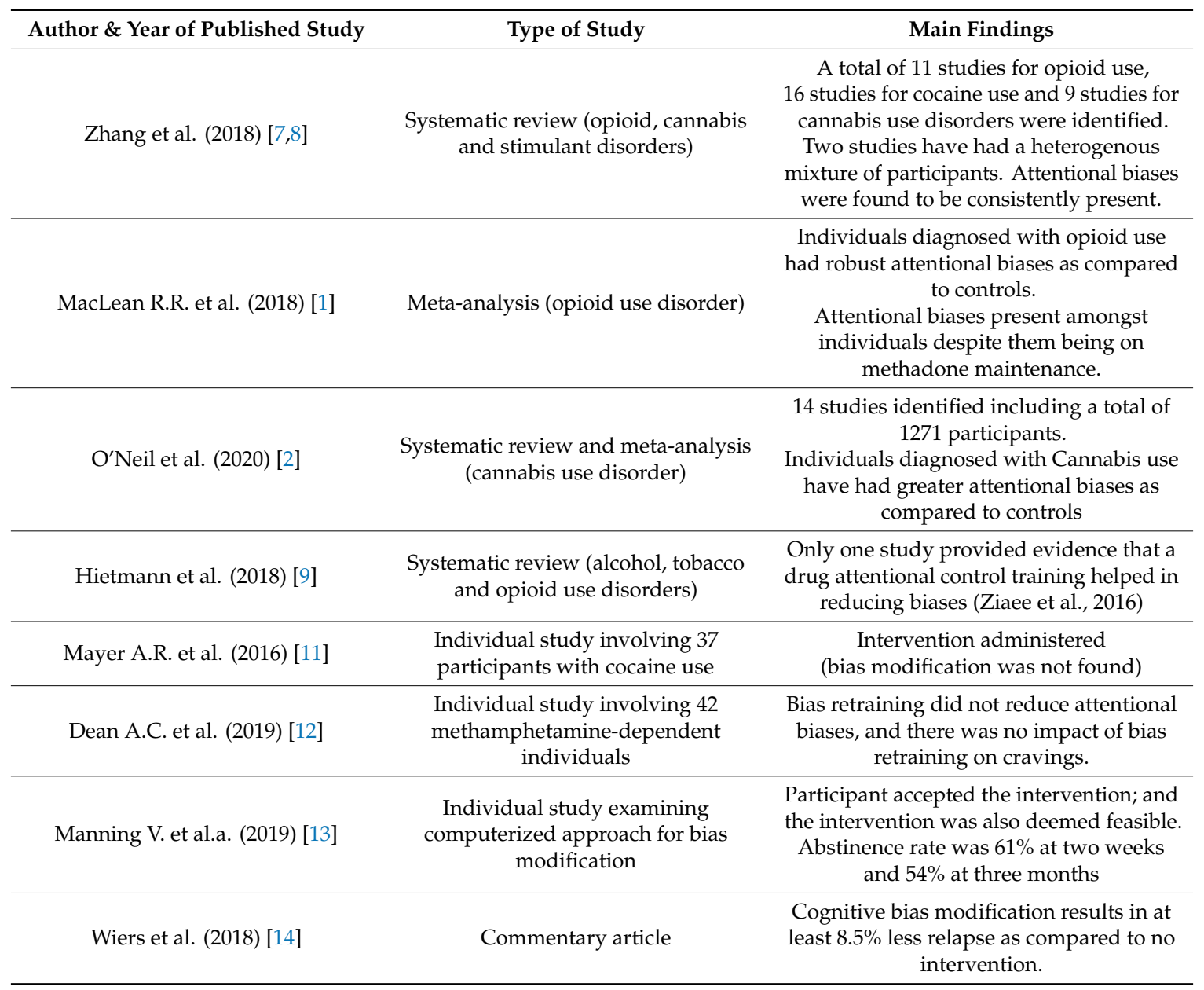

\section{Technological Advances and Its Impact on Bias Modification}

The landscape of the delivery of cognitive bias intervention has evolved with the advances in technologies. Zhang et al. (2019) [15] undertook a scoping review of web-based interventions. Of the 22 published interventions which they have identified, only four studies were for addictive disorders, that of tobacco use and alcohol use disorders. The evidence remained mixed for the four identified studies regarding the effectiveness of web-based bias medication intervention, as there were both trials reporting positive and negative findings. Whilst this has been the case for web-based interventions, this is not the case for mobile cognitive bias modification interventions. In Zhang et al. (2018)'s [16] review of mobile-based cognitive bias modification interventions, they reported there being eight published studies that have harnessed mobile technologies for the delivery of bias modification interventions. Seven out of these eight studies have reported the effectiveness of bias modification, of which three studies were for addictive disorders.

Since the publication of these reviews $[15,16]$, there have been other studies demonstrating the potential of mobile delivery of bias interventions for substance disorders. Most recently, Zhu Y. et al. (2018) [17] described how they evaluated a newly designed computerized cognitive addiction therapy application for individuals with methamphetamine disorders. The tasks included within the application aimed to help eliminate drug-related attentional biases. They reported that whilst the tasks could help improve cognitive impairments and impulse control, there was no significant reduction in attentional biases following the intervention. Since Zhu et al. (2018) [17]'s work, Zhang et al. (2019) [18] have advanced it further by evaluating a conventional mobile attention bias modification intervention amongst individuals with highly prevalent substance use disorders. 
Whilst Zhang et al. (2019)'s [18] prior work was aimed at determining the feasibility and acceptability of the mobile intervention, they found that 14 out of their 30 participants had a change in their baseline biases. Zhang et al. (2020) [19] have recently also described a protocol, that examines if a co-designed gamified attention bias modification intervention was more effective as compared to the conventional intervention. Unfortunately, the authors have not shared any further results. From our knowledge, there remains no other recent study that has examined a mobile approach in the modification of attentional biases for individuals with highly prevalent substance use disorders. Table 2 provides an overview and summarizes the main findings of the included studies.

Table 2. Overview and Summary of Key Characteristics of Included Studies.

\begin{tabular}{ccc}
\hline Author and Year of Published Study & Type of Study & Main Findings \\
Zhang et al. (2019) [15] & $\begin{array}{c}\text { Scoping review of web-based } \\
\text { interventions }\end{array}$ & $\begin{array}{c}\text { Of the 22 identified interventions, } \\
\text { four focused on addictive disorders. } \\
\text { Mixed evidence for the effectiveness } \\
\text { of web-based bias modification for } \\
\text { addictive disorders }\end{array}$ \\
Zhang et al. (2018) [16] & $\begin{array}{c}\text { Review of mobile-based cognitive } \\
\text { bias modification interventions }\end{array}$ & $\begin{array}{c}\text { A total of } 8 \text { studies were identified; } \\
7 \text { out of the } 8 \text { studies reported } \\
\text { effectiveness of bias modification; } \\
\text { of which 3 included participants with } \\
\text { addictive disorders }\end{array}$ \\
Zhu Y. et al. (2018) & $\begin{array}{c}\text { Individual Study describing the } \\
\text { evaluation of a newly designed } \\
\text { computerized cognitive therapy } \\
\text { addiction application for individuals } \\
\text { with methamphetamine disorder }\end{array}$ & $\begin{array}{c}\text { No significant reduction in attentional } \\
\text { biases following the intervention }\end{array}$ \\
Zhang et al. (2019) [18] & $\begin{array}{c}\text { Individual Study, examining the } \\
\text { feasibility and acceptability of } \\
\text { mobile intervention }\end{array}$ & $\begin{array}{c}\text { 14 out of } 30 \text { participants have had a } \\
\text { change in baseline biases }\end{array}$ \\
\hline
\end{tabular}

\section{Biological Underpinnings of Cognitive Biases \& Cognitive Bias Modification}

Zhang et al. (2018) [6], in their previous perspective article, have highlighted the biological basis for attentional biases amongst substance users. They reported how electrophysiological measures had been used to characterize attentional biases, and reported that individuals in abstinence continue to have biases against substance-related cues, with electrophysiological data suggesting there is an overall shorter P1 latency, larger N1, N2 and P2 amplitudes [15]. They also reported the findings of fMRI studies, which have demonstrated that attentional bias is associated with activations in the anterior cingulate cortex, dorsolateral prefrontal cortex, insula, nucleus accumbens and amygdala [13].

More recently, there has been further work done to investigate the brain changes following the administration of attention bias or approach bias modification. Unfortunately, these studies have not reported any positive findings. Mayer A.R. et al. (2020) [20] examined whether attention bias modification affected brain functioning and attempted to characterize brain changes following interventions. They reported that individuals presented with slower reaction times, and increased functional activation in the prefrontal cortex, posterior partial cortex whilst being presented with incongruent versus congruent stimuli, but bias modification did not result in changes in functional activation of the brain. The findings of Karoly H.C. et al. (2019) [21] echoed the previous findings of Mayer A.R. et al. (2020) [20]. They reported that bias modification and training did not alter neural cannabis cue reactivity, as compared to sham experiments.

\section{Challenges with Implementing Cognitive Bias Modification for Substance Disorders}

One of the key challenges with cognitive bias modification pertains to the repetitiveness of the intervention, and the diminished motivation over time. More recently, there has been further investigation in the use of serious games and gamification technologies to reduce the boredom 
associated with these interventions; and potentially to minimize the attrition due to poor motivation to train. Zhang et al. (2018) [22], in their review of gamified cognitive bias modification interventions, reported only two out of four interventions to be effective; these two interventions were for individuals with anxiety disorders. One of the studies by Boendmarker et al. (2016) [23] reported there being no added effectiveness with the integration of gamification elements; despite their previous article suggesting various methods by which gamification could be added onto a conventional task. Zhang et al. (2019) [24], in their work involving the co-design of a mobile attention bias modification, have attempted to seek outpatient and healthcare professionals' perspectives of gamification. In their study, gamification was deemed to be useful in addressing the limitations, and gaming elements such as feedback, levels and rewards were recommended to be considered for future attention bias modification interventions.

\section{Outstanding Gaps in Our Knowledge}

There have been massive advances in cognitive bias modification for individuals with substance use disorders. Cognitive bias modification has conventionally been considered only for individuals with alcohol and tobacco use disorders, but it has been increasingly examined for individuals with highly prevalent substance use disorders, that of opioid use, cannabis use and stimulant use disorders. The increased recognition of the need for cognitive bias modification might be due to there being more evidence to suggest that these biases are consistently present amongst individuals with these disorders. Unfortunately, the evidence for bias modification appears mixed. The lack of effectiveness could potentially be due to the small sample sizes used, and thus leading to there being inadequate power to determine a difference. Moreover, some samples have recruited participants who are in residential detoxification treatment, and it is not clear whether medications administered, or other aspects of the treatment could have attenuated and affected the effectiveness of the intervention, as it is known that certain medications, such as antidepressants and antipsychotics could lead to changes in the magnitude of attentional biases. Future research ought to take into consideration these factors. However, the fact that these interventions have been considered amongst a clinical population implies that there is a potential to consider the clinical implementation of these interventions if there are more definitive results in the future.

Whilst there have been some prior investigations of mobile-based attention bias modification intervention, the research examining this for substance use disorder is still in its infancy. Notably, there has only been two prior evaluations of a mobile-based intervention for substance use disorders: those of Zhang et al. (2019) and Zhu Y. et al. (2018) [17]. Whilst Zhu Y. et al. (2018)'s [23] study did not report any positive biases pertaining to bias modification training, it must be noted that the authors have not based their training on a conventional paradigm, but have adapted it. Meanwhile Zhang et al. (2019)'s [18] study highlighted the potential and acceptability of mobile delivery of these interventions, but in their subsequent research, also highlighted issues pertaining to a conventional mobile bias intervention. Future research should consider further evaluative work harnessing the potential of mobile technologies for the delivery of bias intervention; furthermore, future studies should seek to determine the effectiveness of such interventions amongst varied substance use conditions. There might also be a need for future research to consider a co-design approach, as Zhang et al. (2019) [24] have highlighted how the adoption of such an approach could resolve some of the inherent limitations of the application, and also potentially considering the inclusion of other elements that could help with motivation to train, with a resultant reduction in attrition.

Lastly, it might still be worthwhile to examine the dynamic changes in brain functioning and connectivity of the neural circuit before and after bias modification intervention, despite the recent negative findings, as there is evidence suggesting that there are dynamic changes in the brain when attentional biases are present. 


\section{Conclusions}

The discovery of automatic, unconscious biases amongst individuals with addictive disorders previously led to further investigation of these biases, and the modification of these biases in substance-using individuals. This perspective article has mapped out the advances and new knowledge about bias assessment and modification amongst the highly prevalent substance use disorders and provided a synopsis of the advances in the delivery of these interventions, and an overview of neurobiological research findings. This perspective article has also highlighted the main challenge involving the administration of these bias modification tasks. There remain several gaps in the knowledge that we hope that future research could address.

Author Contributions: M.W.B.Z. and H.E.S. jointly conceptualized the study. M.W.B.Z. wrote up the first draft of the manuscript, with guidance from H.E.S. All authors have read and agreed to the published version of the manuscript.

Funding: MWZ is supported by a grant under the Singapore Ministry of Health's National Medical Research Council (grant number NMRC/Fellowship/0048/2017) for PhD training. The funding source was not involved in any part of this project.

Conflicts of Interest: The authors declare no conflict of interest.

\section{References}

1. MacLean, R.R.; Sofuoglu, M.; Brede, E.; Robinson, C.; Waters, A.J. Attentional bias in opioid users: A systematic review and meta-analysis. Drug Alcohol Depend. 2018, 191, 270-278. [CrossRef] [PubMed]

2. O'Neill, A.; Bachi, B.; Bhattacharyya, S. Attentional bias towards cannabis cues in cannabis users: A systematic review and meta-analysis. Drug Alcohol Depend. 2020, 206, 107719. [CrossRef] [PubMed]

3. Cristea, I.A.; Kok, R.N.; Cuijpers, P. The effectiveness of cognitive bias modification interventions for substance addictions: A meta-analysis. PLoS ONE 2016, 11, e0162226. [CrossRef] [PubMed]

4. Boffo, M.; Zerhouni, O.; Gronau, Q.F.; Van Beek, R.J.J.; Nikolaou, K.; Marsman, M.; Wiers, R.W. Cognitive bias modification for behavior change in alcohol and smoking addiction: Bayesian meta-analysis of individual participant data. Neuropsychol. Rev. 2019, 29, 52-78. [CrossRef] [PubMed]

5. United Nations Office on Drug and Crime. Available online: https://www.unodc.org/unodc/en/data-andanalysis/statistics/index.html (accessed on 18 September 2020).

6. Zhang, M.W.; Ying, J.B.; Song, G.; Fung, D.S.S.; Smith, H.E. Recent advances in attention bias modification for substance addictions. Int. J. Environ. Res. Public Health 2018, 15, 676. [CrossRef] [PubMed]

7. Zhang, M.W.B.; Ying, J.; Wing, T.; Song, G.; Fung, D.S.S.; Smith, H.E. Cognitive biases in cannabis, opioid, and stimulant disorders: A systematic review. Front. Psychiatry 2018, 9, 376. [CrossRef]

8. Zhang, M.; Ying, J.; Wing, T.; Song, G.; Fung, D.S.S.; Smith, H. A systematic review of attention biases in opioid, cannabis, stimulant use disorders. Int. J. Environ. Res. Public Health 2018, 15, 1138. [CrossRef]

9. Heitmann, J.; Bennik, E.C.; Van Hemel-Ruiter, M.E.; De Jong, P.J. The effectiveness of attentional bias modification for substance use disorder symptoms in adults: A systematic review. Syst. Rev. 2018, 7, 1-21. [CrossRef]

10. Ziaee, S.S.; Fadardi, J.S.; Cox, W.M.; Yazdi, S.A.A. Effects of attention control training on drug abusers' attentional bias and treatment outcome. J. Consult. Clin. Psychol. 2016, 84, 861-873. [CrossRef]

11. Mayer, A.R.; Wilcox, C.E.; Dodd, A.B.; Klimaj, S.D.; Dekonenko, C.J.; Claus, E.D.; Bogenschutz, M. The efficacy of attention bias modification therapy in cocaine use disorders. Am. J. Drug Alcohol Abus. 2016, 42, 459-468. [CrossRef]

12. Dean, A.C.; Nurmi, E.L.; Moeller, S.J.; Amir, N.; Rozenman, M.; Ghahremani, D.G.; Johnson, M.; Berberyan, R.; Hellemann, G.; Zhang, Z.; et al. No effect of attentional bias modification training in methamphetamine users receiving residential treatment. Psychopharmacology 2018, 236, 709-721. [CrossRef] [PubMed]

13. Manning, V.; Garfield, J.B.B.; Mroz, K.; Campbell, S.C.; Piercy, H.; Staiger, P.K.; Lum, J.A.G.; Lubman, D.I.; Verdejo-Garcia, A. Feasibility and acceptability of approach bias modification during methamphetamine withdrawal and related methamphetamine use outcomes. J. Subst. Abus. Treat. 2019, 106, 12-18. [CrossRef] 
14. Wiers, R.W.; Boffo, M.; Field, M. What's in a Trial? The authors respond: Persistent mixing of apples and oranges, or carefully synthesizing and designing the next steps in research on cognitive bias modification in addiction. J. Stud. Alcohol Drugs 2018, 79, 348-349. [CrossRef] [PubMed]

15. Zhang, M.; Ying, J.; Song, G.; Fung, D.S.S.; Smith, H. Web-based cognitive bias modification interventions for psychiatric disorders: Scoping review. JMIR Ment. Health 2019, 6, e11841. [CrossRef]

16. Zhang, M.; Ying, J.; Song, G.; Fung, D.S.S.; Smith, H. Attention and cognitive bias modification apps: Review of the literature and of commercially available apps. JMIR mHealth uHealth 2018, 6, e10034. [CrossRef]

17. Zhu, Y.; Jiang, H.; Su, H.; Zhong, N.; Li, R.; Li, X.; Chen, T.; Tan, H.; Du, J.; Xu, D.; et al. A newly designed mobile-based computerized cognitive addiction therapy app for the improvement of cognition impairments and risk decision making in methamphetamine use disorder: Randomized controlled trial. JMIR mHealth uHealth 2018, 6, e10292. [CrossRef] [PubMed]

18. Zhang, M.; Ying, J.; Amron, S.B.; Mahreen, Z.; Song, G.; Fung, D.S.S.; Smith, H.E. A smartphone attention bias app for individuals with addictive disorders: Feasibility and acceptability study. JMIR mHealth uHealth 2019, 7, e15465. [CrossRef] [PubMed]

19. Zhang, M.W.B.; Heng, S.; Amron, S.B.; Mahreen, Z.; Song, G.; Fung, D.S.S.; Smith, H.E. Gamified m-health attention bias modification intervention for individuals with opioid use disorder: Protocol for a pilot randomised study. Int. J. Environ. Res. Public Health 2020, 17, 752. [CrossRef] [PubMed]

20. Mayer, A.R.; Dodd, A.B.; Wilcox, C.E.; Klimaj, S.D.; Claus, E.D.; Bryan, A.D. Effects of attentional bias modification therapy on the cue reactivity and cognitive control networks in participants with cocaine use disorders. Am. J. Drug Alcohol Abus. 2019, 46, 357-367. [CrossRef] [PubMed]

21. Karoly, H.C.; Schacht, J.P.; Jacobus, J.; Meredith, L.R.; Taylor, C.T.; Tapert, S.F.; Gray, K.M.; Squeglia, L.M. Preliminary evidence that computerized approach avoidance training is not associated with changes in $\mathrm{fMRI}$ cannabis cue reactivity in non-treatment-seeking adolescent cannabis users. Drug Alcohol Depend. 2019, 200, 145-152. [CrossRef] [PubMed]

22. Zhang, M.; Ying, J.; Song, G.; Fung, D.S.; Smith, H. Gamified cognitive bias modification interventions for psychiatric disorders: Review. JMIR Ment. Health 2018, 5, e11640. [CrossRef] [PubMed]

23. Boendermaker, W.J.; Maceiras, S.S.; Boffo, M.; Wiers, R.W. Attentional bias modification with serious game elements: Evaluating the shots game. JMIR Serious Games 2016, 4, e20. [CrossRef]

24. Zhang, M.; Heng, S.; Song, G.; Fung, D.S.S.; Smith, H.E. Co-designing a mobile gamified attention bias modification intervention for substance use disorders: Participatory research study. JMIR $m$ Health $u$ Health 2019, 7, e15871. [CrossRef]

Publisher's Note: MDPI stays neutral with regard to jurisdictional claims in published maps and institutional affiliations.

(C) 2020 by the authors. Licensee MDPI, Basel, Switzerland. This article is an open access article distributed under the terms and conditions of the Creative Commons Attribution (CC BY) license (http://creativecommons.org/licenses/by/4.0/). 